ARTICLE

\title{
Structure-controllable growth of nitrogenated graphene quantum dots via solvent catalysis for selective $\mathrm{C}-\mathrm{N}$ bond activation
}

\author{
Byung Joon Moon ${ }^{1,2,7}$, Sang Jin Kim,7, Aram Lee ${ }^{1,7}$, Yelin Oh', Seoung-Ki Lee ${ }^{3}$, Sang Hyun Lee ${ }^{4}{ }^{4}$, \\ Tae-Wook Kim (10 ${ }^{5}$, Byung Hee Hong ${ }^{2,6}$ \& Sukang Bae (i) ${ }^{1 凶}$
}

Photophysical and photochemical properties of graphene quantum dots (GQDs) strongly depend on their morphological and chemical features. However, systematic and uniform manipulation of the chemical structures of GQDs remains challenging due to the difficulty in simultaneous control of competitive reactions, i.e., growth and doping, and the complicated post-purification processes. Here, we report an efficient and scalable production of chemically tailored N-doped GQDs (NGs) with high uniformity and crystallinity via a simple onestep solvent catalytic reaction for the thermolytic self-assembly of molecular precursors. We find that the graphitization of $\mathrm{N}$-containing precursors during the formation of NGs can be modulated by intermolecular interaction with solvent molecules, the mechanism of wh ich is evidenced by theoretical calculations and various spectroscopic analyses. Given with the excellent visible-light photoresponse and photocatalytic activity of NGs, it is expected that the proposed approach will promote the practical utilization of GQDs for various applications in the near future.

\footnotetext{
${ }^{1}$ Functional Composite Materials Research Center, Korea Institute of Science and Technology, Wanju-gun, Jeollabuk-do 55324, Republic of Korea.

2 Department of Chemistry, Seoul National University, Seoul 08826, Republic of Korea. ${ }^{3}$ School of Materials Science and Engineering, Pusan National University, Busan 46241, Republic of Korea. ${ }^{4}$ The School of Chemical Engineering, Chonnam National University, Gwangju 61186, Republic of Korea.

${ }^{5}$ Department of Flexible and Printable Electronics, Jeonbuk National University, Jeonju-si, Jeollabuk-do 54896, Republic of Korea. ${ }^{6}$ Graphene Research Center, Advanced Institute of Convergence Technology, Seoul National University, Suwon-si, Gyeonggi-do 16229, Republic of Korea. ${ }^{7}$ These authors contributed equally: Byung Joon Moon, Sang Jin Kim and Aram Lee. 凶email: sbae@kist.re.kr
} 
G raphene quantum dots (GQDs) are a relatively new class of low-cost carbon-based fluorescent nanomaterials that have been recognized as great potential candidates for various research areas of photonics and biochemistry and applications for catalysis, energy conversion/storage, and sensing owing to their unique chemical, electrochemical, optical, and biological properties ${ }^{1-3}$. To be appreciated as a practical alternative to the previously used materials in numerous applications, the chemical composition or configuration of GQDs should be systematically manipulated, as these features are closely related to the biocompatibility, catalytic behaviour, chemical reactivity/ selectivity, and optical responses, e.g., emission dynamics, absorption/emission window, and photosensitivity, of the materials ${ }^{4-7}$. In an attempt to tailor the chemical features of GQDs, substitution of heteroatoms into the graphitic lattice and the introduction of specific chemical functional groups to the edge sites have been actively studied ${ }^{4-6,8}$. Recently, as a simple but efficient "bottom-up" method, one-pot pyrolysis using single or multiple organic precursors has been widely utilized to prepare heteroatom-doped GQDs with lateral sizes ranging from 1 to $10 \mathrm{~nm}$ due to the simplicity of the process ${ }^{8-13}$. Additionally, some reports have revealed the feasibility of obtaining edge-controlled GQDs by changing the types of solvents used during the formation processes ${ }^{14,15}$.

Despite these early successes in the production of heteroatomdoped GQDs, there still exist many challenges in the optimization of fabrication processes, including the removal of acidic/basic intermediates and dopant residues ${ }^{11,16-19}$, which necessarily involve time/cost-consuming post-treatments and hinder largescale manufacturing. Furthermore, current techniques derived from solution-based one-pot methods are insufficient to allow simultaneous control of both the structural features (crystallinity) and doping characteristics (concentrations and configurations). Essentially, this limitation is caused by either the amorphous structure of dopants or the incompatibility with thermolysis because of the high volatility of dopants and the resulting increased internal pressure of the reaction system. Moreover, the uncontrollable kinetics of subsequent competitive reactions, such as growth of graphitic domains and additive (dopant)-induced side reactions that form molecular fluorophores, may impede an intuitive prediction of the synthesis $10,16,20,21$.

Additionally, further development of such synthetic strategies towards diverse applications has been hindered by the absence of detailed investigations into the correlation between optical properties and the surface status of doped GQDs, which is passivated by the incomplete carbonization of organic precursors $^{11,22}$.

Furthermore, in the field of "traditional" organic synthesis, numerous studies have proved the crucial role of solvent parameters (hereafter referred to as "solvent effect"), including the dielectric constant, polarity, and basicity. Taking one step further, studies on the perspective of reaction kinetics and selectivity have revealed that the solvent influences on the reaction velocity, which may be described as a catalytic effect, and govern the transformation of various functional groups in organic molecules ${ }^{23,24}$. Unfortunately, despite its criticality, there have been only a few studies connecting this effect to the chemical modification of GQDs, which may reveal the mechanism of the doping profile changes in response to the solvent-assisted pyrolysis of organic precursors, e.g., glucose and citric/glutamic acids.

In this work, we report a single molecular precursor-based synthetic platform to synthesize chemically tailored $\mathrm{N}$-doped graphene quantum dots (NGs) with high crystallinity and homogeneity that are self-passivated by various functional moieties with different chain lengths. Such an approach could provide a predictable synthetic tool for solvent-catalysts-aided growth of heteroatom-doped GQDs via selective and quantitative $\mathrm{C}-\mathrm{N}$ bond activation. By combining experimental results with quantum chemical calculations, we determine the reaction mechanism of oxidative activation of the nitrile groups in fumaronitrile (FN) in a variety of polar solvents, as well as the relationship between the structural features and optical characteristics of NGs using both steady-state and time-dependent density functional theory (SSand TD-DFT, respectively), which can reveal the effects of end moieties as well as $\pi$-linkers. Finally, we conclude our study by exploring one of its practical applications, namely, light-sensitized photocatalysts. Specifically, to investigate the structural effects of NGs, we first apply GQDs for photocatalytic wastewater treatment, and more importantly, their potential medical application in ultralow-level energy photodynamic therapy (UL-PDT) is also validated. Consequently, the observed differences in the photocatalytic activities of each NG prepared from different solvent systems are consistent with our pre-estimations, intuitively reconfirming the effectiveness of our fabrication approach and the significance of theoretical prediction from both chemical and optical perspectives.

\section{Results and Discussion}

Synthesis and chemical characterization of NGs. In our study, NGs were synthesized by a one-step self-assembly growth method in which the organic precursor FN was added to each of the following four different solvent media: DI water (DW), THF, butanal $(\mathrm{Bu})$, and benzaldehyde $(\mathrm{Be})$. As graphically summarized in Fig. 1, the complete self-assembly reaction of FN proceeds via two distinctive but simultaneous processes. Most of the primary reactant in $\mathrm{FN}$ hydrolysis, i.e., $\mathrm{H}_{2} \mathrm{O}$, is assumed to be supplied from moisture in the air within the reaction chamber, which is actively diffused or permeated into the reaction volume by increased pressure at high reaction temperatures. More detailed descriptions are provided in the Supplementary Information (see Supplementary Figs. 1-5 and Supplementary Discussion 1).

First, to confirm the variations in chemical composition and bonding of each NG under different solvent effects, X-ray photoelectron spectroscopic (XPS) and Fourier transforminfrared spectroscopic (FT-IR) measurements were performed (Fig. 2a-c; Supplementary Figs. 6-9). According to the XPS results in Fig. 2a, the change in the $\mathrm{O} / \mathrm{C}$ atomic ratio of NGs follows a trend opposite to that for the N/C value (top of Fig. 2c). In addition, in terms of the chemical bonding ratios of the NGs, there is an inverse correlation between the concentration of oxidized $\mathrm{C}$ and that of nitrogenated $\mathrm{C}$ (see Fig. $2 \mathrm{~b}$ and bottom panel in 2c), which was also confirmed by the FT-IR data (Supplementary Fig. 9). The chemical structures of two representative NGs (NG_DW and NG_Be: most and least oxidized forms, respectively) are depicted in Fig. 2e. Because of the differences in functional moieties of each QD, their $\mathrm{pH}$ values were found to be slightly different, as shown in Supplementary Fig. 10. These analyses show the feasibility of effectively modulating the chemical bonding configuration and composition of NGs during the thermolytic self-assembly reaction of FN by applying different solvent conditions.

Theoretical study on the chemical activation of FN. To theoretically support these experimental observations of variations in the chemical composition of NGs, we performed DFT calculations by using the Gaussian 16 program package (Fig. $2 \mathrm{~d}$ and $\mathrm{f}$ ). Complete chemical conversion of FN proceeds in two consecutive steps (Step 1: $\mathrm{C} \equiv \mathrm{N} \rightarrow \mathrm{CONH}_{2}$ and Step 2: $\mathrm{CONH}_{2} \rightarrow \mathrm{COOH}$ ), as depicted in Supplementary Fig. 11. First, the two most plausible mechanisms for the $\mathrm{C}-\mathrm{N}$ activation of $\mathrm{FN}(\mathrm{FN} \rightarrow \mathrm{FAm}$ (fumaramide-like molecule)) are depicted at the top of Fig. $2 \mathrm{~d}$, 


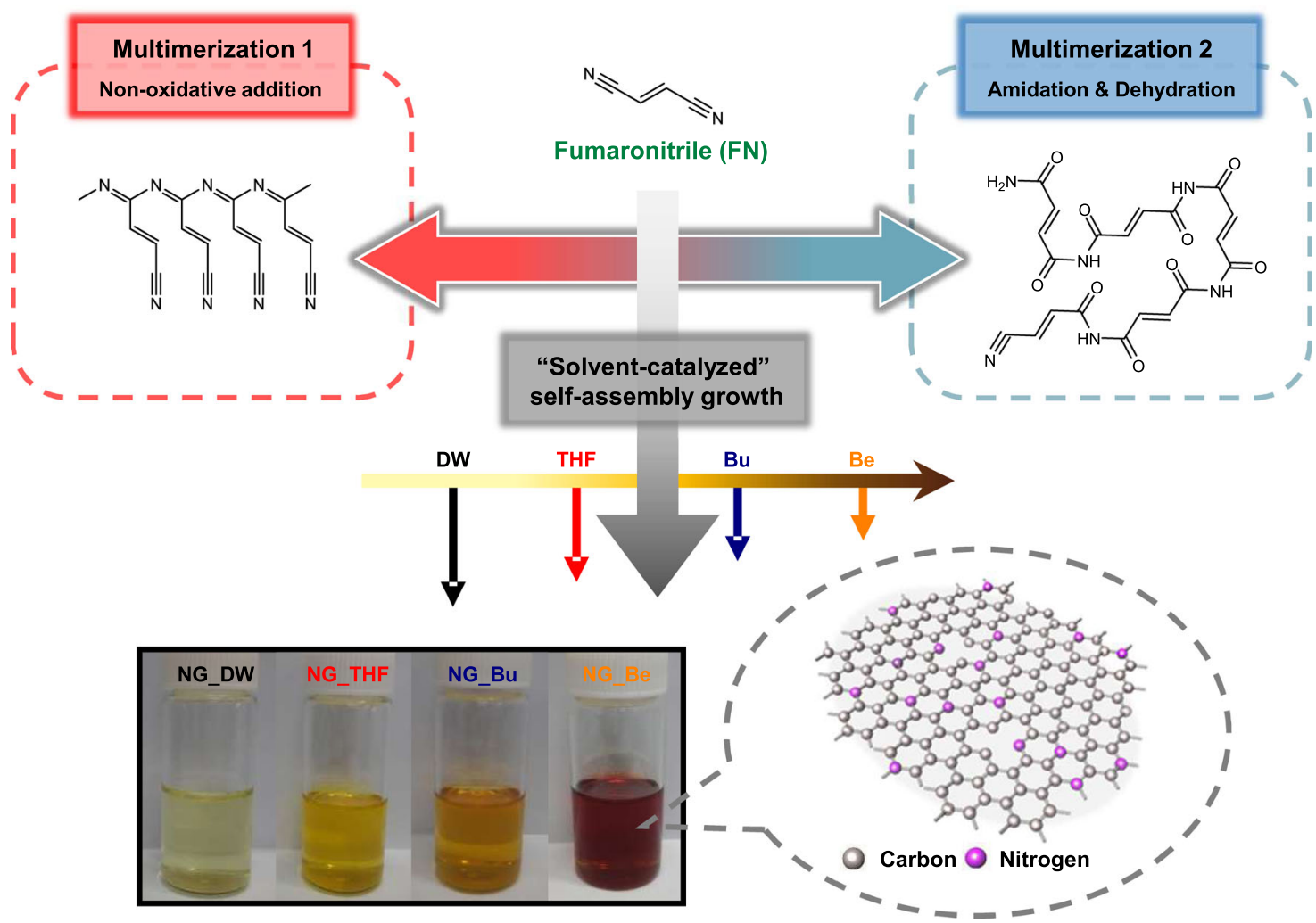

Fig. 1 Illustration of the formation mechanism of chemically-tailored NGs. Schematic diagram of the possible formation mechanisms of NGs through the thermolytic self-assembly reaction of $\mathrm{FN}$, and photographs of their ethanol solution preparation under four different solvent systems.

where Paths I and II represent the general solvolysis reaction and solvent-catalysed oxidation reaction, respectively. Both transformation mechanisms of the $\mathrm{C} \equiv \mathrm{N}$ functional group are fundamentally based on the addition-elimination mechanism, adding nucleophile molecules to electron-deficient $\mathrm{C}$ atoms.

From the thermodynamic numerical analyses in the Fig. $2 d$, the supporting solvent molecules (SSMs) in our study, including THF, $\mathrm{Bu}$, and $\mathrm{Be}$, were eliminated from the candidates of nucleophiles due to their low affinity to the electron-deficient $\mathrm{C}$ atoms. Furthermore, based on the free energy calculations in Supplementary Fig. 12a, oxidative activation of FN would rarely be achieved in circumstances where the SSMs intervened in the net reaction and the barrier energy was accordingly increased. Therefore, those SSMs were ruled out from the potential causes of the high crystallinity in our NGs, represented with a transmission electron microscopy (TEM) image in Fig. 3b.

Alternatively, as shown in Supplementary Fig. 12b, all SSMs more likely serve themselves as pseudo-electron-withdrawing moieties for the $\mathrm{C} \equiv \mathrm{N}$ group in the reaction system $(\Delta H<0)$ and generate a cation state $\left(\mathrm{C} \equiv \mathrm{N}^{+}\right)$. In other words, this result suggests that the solvent used is not only the medium where the chemical reactions are performed, but it also actively involves in the chemical process. Furthermore, the change in Gibbs free energy $(\Delta G)$ for this step is positive due to the negative contribution of the entropy change caused by the formation of $\mathrm{H}$-bonds and increased van der Waals forces.

More importantly, the overall profiles of the potential energy surface of FN activation depend significantly on the types of solvents. In other words, as shown in the left of Fig. $2 \mathrm{f}$, the change in $\Delta G$ is quite different in every elementary reaction for the transformation of the $-\mathrm{C} \equiv \mathrm{N}$ group to its oxidized form. In particular, it is clearly observed that there is a distinct difference in the $\Delta G$ value of the rate-determining region (intermediate state structure \#2 (IM2) - transition state structure \#1 (TR1) \& IM6
- TR2) in each reaction step. From the theoretical estimation of $60 \%$ higher activation barrier for TR1_Be than for TR1_DW, the corresponding pathway is considered relatively improbable and is simultaneously suppressed, regardless of its thermodynamic energy. The above thermodynamic and kinetic calculations signify that the SSMs can effectively control the oxidation degree of FN. Consequently, we can argue that the solvent in the reaction system does not only serve as a dispersing agent, but also it can be promoted as an active interventor which can alter the energy characteristics of the system during the intermediate and transition states of the synthesis.

Interestingly, it was found that the values of effective Gibbs free energy $\left(\Delta G_{\text {eff }}\right.$, integration of $\Delta G$ provided in Fig. $\left.2 \mathrm{~d}, \mathrm{f}\right)$ is observed to be a monotonic increasing function of the atomic ratio of resulting NGs (Fig. 2a), and thus this trend can be fitted by a bounded exponential growth curve as plotted in Fig. $2 \mathrm{~g}$. Reminding the importance of $\mathrm{C} \equiv \mathrm{N}$ bond activation in either FN or its multimeric form for the formation of NGs, this correlation seems to be reasonable because their composition modulation can be attributed to the oxidation degree of FN. Also, we investigated the reliability and predictability of the model for the chemical composition of NGs prepared under other solvent systems (Supplementary Fig. 13), proving the universality and effectiveness of our approach for the production of chemically tailored GQDs. Therefore, based on the results obtained from analytical experiments and DFT calculations in this study, we are able to assert that our approach can be used as a predictable synthetic tool for application-specific NGs.

Surface and structural analysis of NGs. For further experimental verifications of the resulting NGs under different solvent effects, the surface morphologies of each sample were investigated by atomic force microscopy (AFM) and TEM. As seen from the 
a

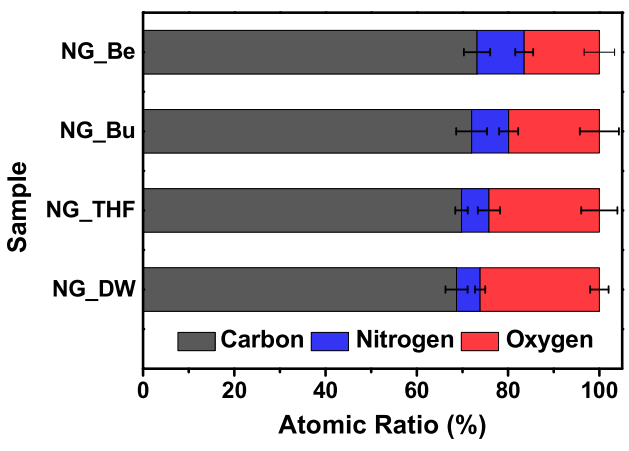

C
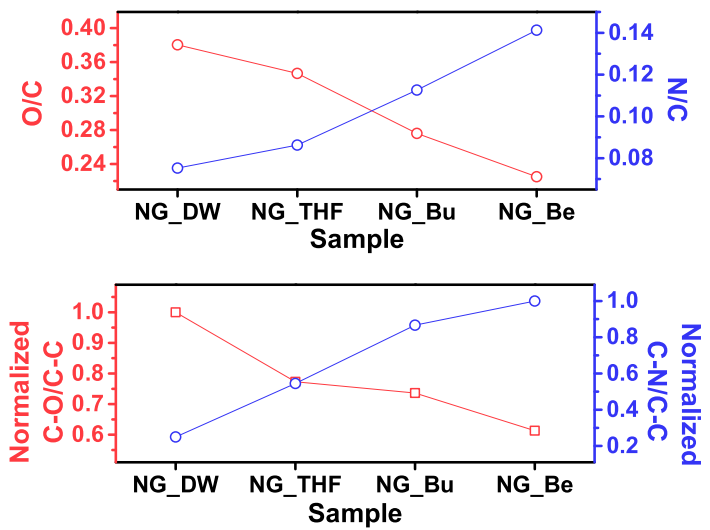

e

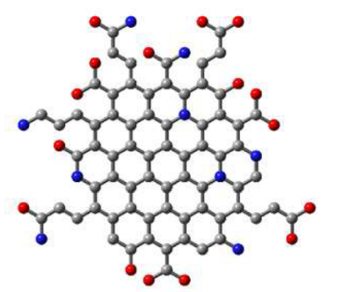

NG_DW

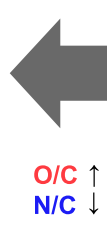

O/C $\uparrow$

$\mathrm{N} / \mathrm{C} \downarrow$

$\mathbf{f}$ b

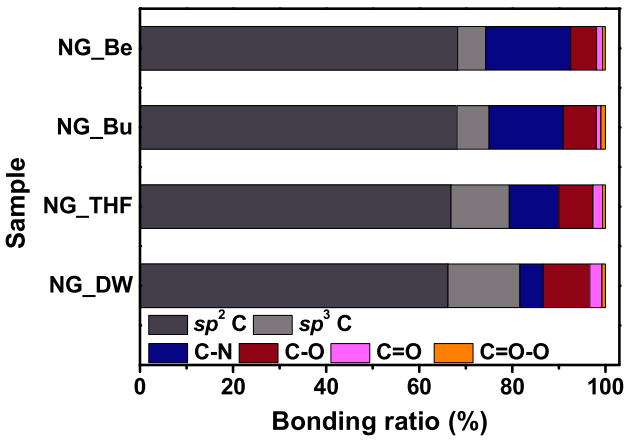

d
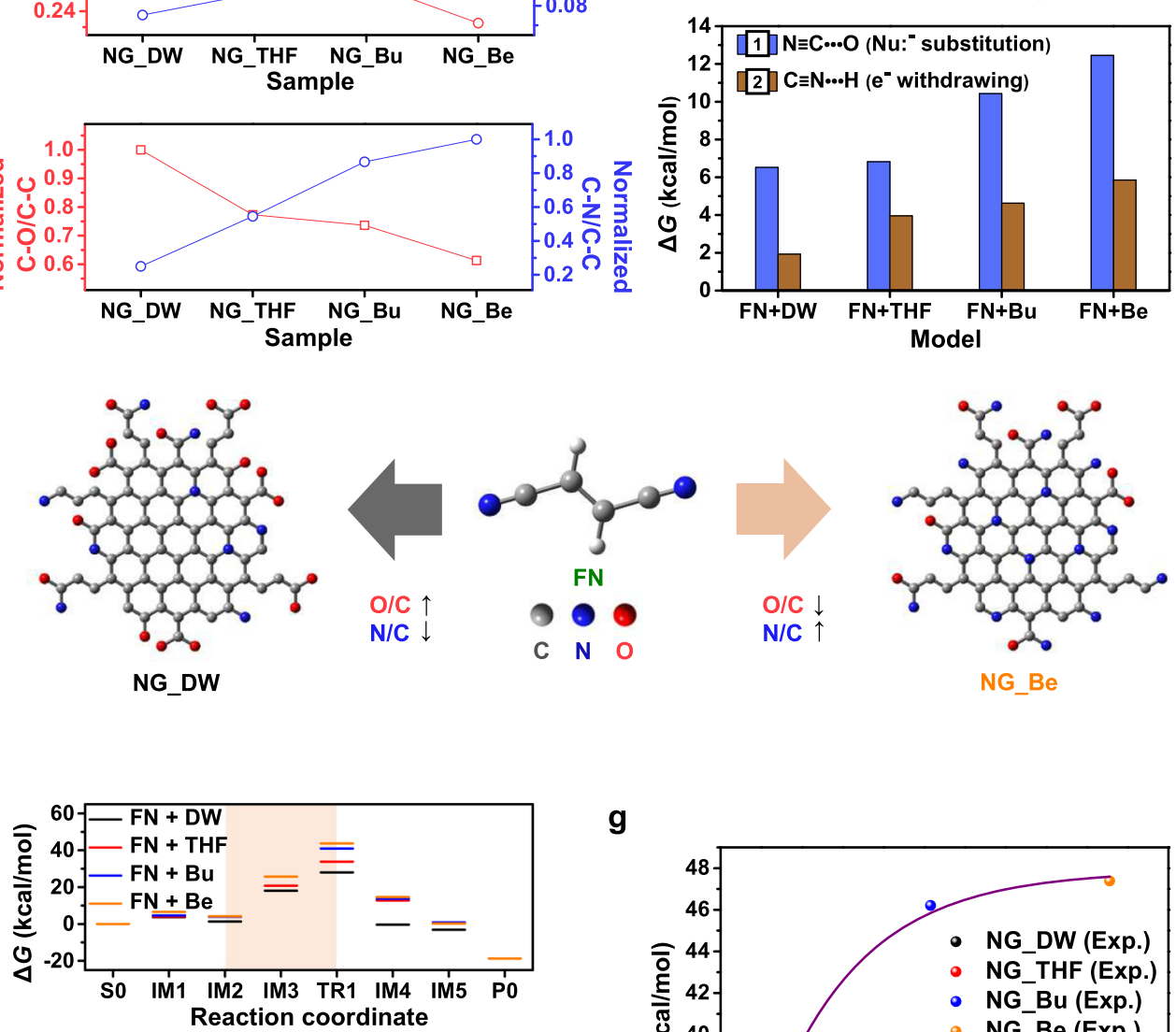

$\mathbf{g}$
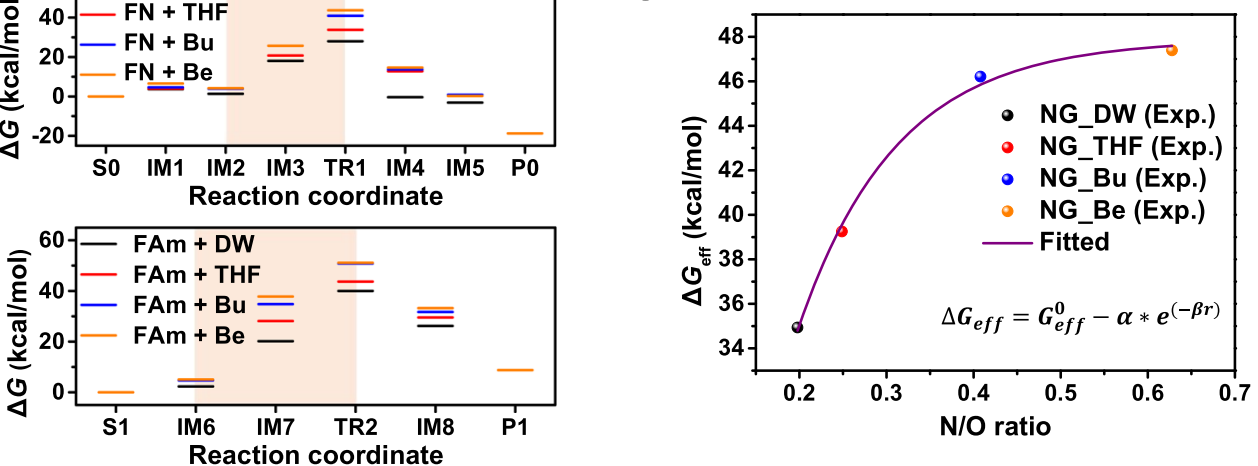

Fig. 2 Chemical composition characterization of NGs. a Elemental analysis from XPS measurements of NGs prepared with different reaction solvents. Error bars represent standard deviations from five independent experiments. b Multiple C-bonding configurations observed in the $\mathrm{C} 1 \mathrm{~s}$ spectra of each NGs. c Relative atomic ratios (O/C and N/C, top) and chemical bonding ratios of NGs (C-N/C-C \& C-O/C-C, bottom). d Computationally derived interaction energies between FN and organic solvent molecules. e Plausible chemical structures of NGs prepared from different solvent systems. $\mathbf{f}$ Free energy profiles for the oxidative activation of FN (top) and FAm (bottom) calculated at the B3LYP/6-311 + G(d) level. $\mathbf{g}$ Correlation between the effective Gibbs free energy $\left(\Delta G_{\text {eff }}\right.$ ), obtained from a weighted average of $\Delta G$ for selected steps, and the atomic ratio of resulting NGs $(\alpha=75.6468 \mathrm{kcal} / \mathrm{mol}$, $\beta=8.8652, G_{\text {eff }}^{0}=47.8776 \mathrm{kcal} / \mathrm{mol}, r=\mathrm{N} / \mathrm{O}$ ratio). 
a

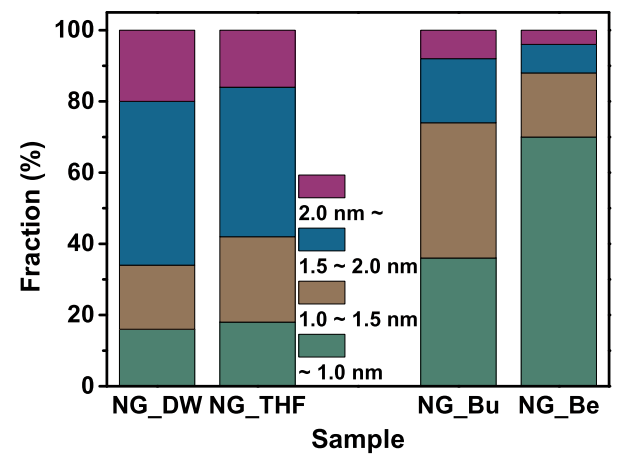

C

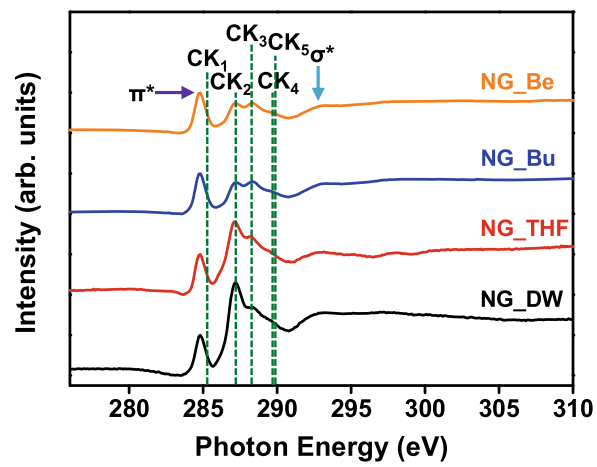

b

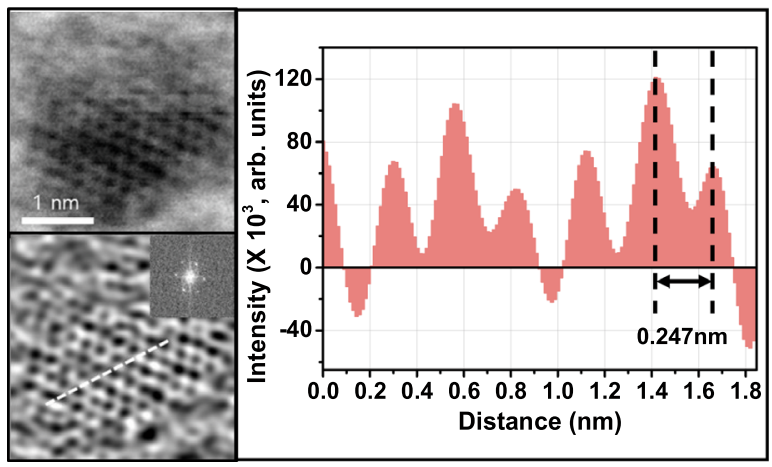

d

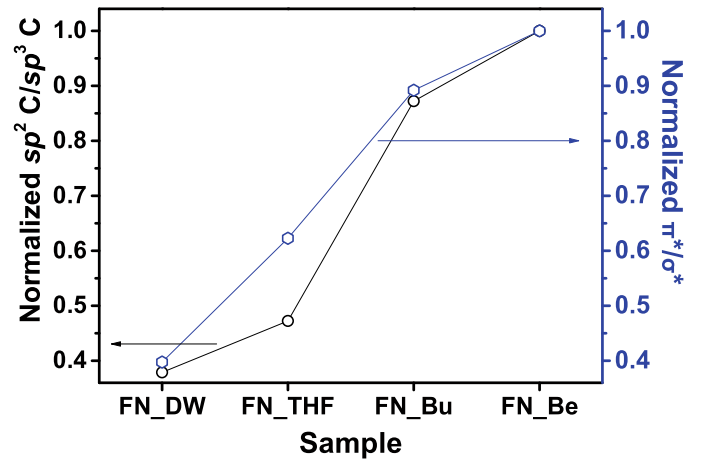

Fig. 3 Morphological and structural characterizations of NGs. a AFM-measured height distributions of NGs (collected at 50 dots per each sample). b Representative STEM image (top-left), corresponding IFFT image of a selected area (bottom-left, inset: FFT image), and intensity line profile (right) along the dashed line of NG_Be (atom distance of $\sim 0.24 \mathrm{~nm}$, matching with average distances in graphitic materials (1120) ${ }^{25}$. c NEXAFS spectra of four different types of NGs. The lowest energy resonance, at $\sim 285.2 \mathrm{eV}$, corresponds to the transition of $\mathrm{C} 1 \mathrm{~s}$ core-level electrons into states of $\pi^{\star}$ symmetry around the $\mathrm{M}$ and $\mathrm{L}$ points of the graphene Brillouin zone above the Fermi level, whereas the higher energy peak at $\sim 293.2 \mathrm{eV}$ can be attributed to the excitation of $\mathrm{C}$ $1 \mathrm{~s}$ core electrons into dispersionless states of $\sigma^{\star}$ symmetry 46,47 . A more detail description is presented in the Method section. $\mathbf{d}$ Ratios of $s p^{2} \mathrm{C}-\mathrm{C} / \mathrm{sp}^{3} \mathrm{C}-\mathrm{C}$ (black circle) and $I_{\sigma^{\star}} / I_{\pi^{*}}$ (blue hexagon) in NGs prepared in different reaction solvents.

AFM images (Supplementary Fig. 20), the heights of all samples range from 0.5 to $2 \mathrm{~nm}$, suggesting the successful synthesis of NGs in all four different reaction conditions. Notably, the average height of NG_Be is found to be $<1.0 \mathrm{~nm}$ (1-2 layers) in Fig. 3a, suggesting effectively suppressed vertical growth compared to the others. At this stage, we suppose that these features are likely related to the competitive reaction pathways for non-oxidative addition multimerization and solvent-assisted oxidation of FN (Supplementary Fig. 22). More discussion regarding the MG growth mechanism is provided in the Supplementary Information (see "Supplementary Discussion 2"). More analyses of the exact origin of the morphological variation are ongoing and will be included in our future studies.

The majority of NG_Be forms a monolayered graphene sheet, and its hexagonal lattice structure was readily observed by means of atomic-resolution scanning transmission electron microscopy (STEM), as displayed at the top left of Fig. 3b and Supplementary Fig. 21. From these data, it is clear that the formed zerodimensional nanostructures are almost defect-free and highly crystalline, with diameters of $<5 \mathrm{~nm}$. These findings are also confirmed by the results of XPS and near-edge X-ray absorption fine structure (NEXAFS) analysis shown in Fig. $3 c$ and $d$, respectively. In particular, the relative intensities of $\pi^{*}$ resonances $\left(\pi^{*} / \sigma^{*}\right)$ in Fig. $3 \mathrm{~d}$ provide an accurate estimate of the fraction of $s p^{2}$ (graphitic region) to $s p^{3}$ (amorphous defect sites) domain configurations, consistent with the XPS results ${ }^{25}$. Additional descriptions of the detailed investigation of the graphitization mechanism are given in the Supporting Information (see “Supplementary Discussion 2").

Experimental and theoretical studies on the optical properties of NGs. To understand the changes in the optical properties of NGs as a result of the solvent effects, UV-visible absorption spectra were obtained, as shown in Fig. 4a (top) and Supplementary Fig. 23a. In addition to the two excitonic absorption bands (described in the caption of Fig. 4a), broad absorption peaks in the relatively lower energy band $(350-500 \mathrm{~nm}$ ) are also observed. These features are reflected in their photoluminescence excitation (PLE) spectra (Supplementary Fig. 23b) as well and consist of two electronic transitions at $260-270 \mathrm{~nm}\left(\pi \rightarrow \pi^{*}\right.$ excitation) and $360-380 \mathrm{~nm}\left(\mathrm{n} \rightarrow \pi^{*} \text { excitation }\right)^{8}$.

In a theoretical determination of the correlation between the molecular structure and optical properties of the NGs, both SSand TD-DFT calculations were performed by following the detailed procedure described in the Method section. The calculated absorption spectra of pristine and modified $\mathrm{C}_{7}-\mathrm{GQDs}$ in ethanol (solvation model: SMD) are presented in Supplementary Fig. 14. Additionally, the calculated parameters of absorption energy, wavelength, and oscillator strength $(f)$ for the first 5 lowest excited states of $\mathrm{C}_{7}$-GQDs are listed in Supplementary Table 1. First, regardless of the types of heteroatom-containing substituents, all $\mathrm{C}_{7}$-GQDs under consideration exhibited a redshift in their absorption wavelengths and much broader FWHMs than the pristine sample, as shown in Supplementary 
a

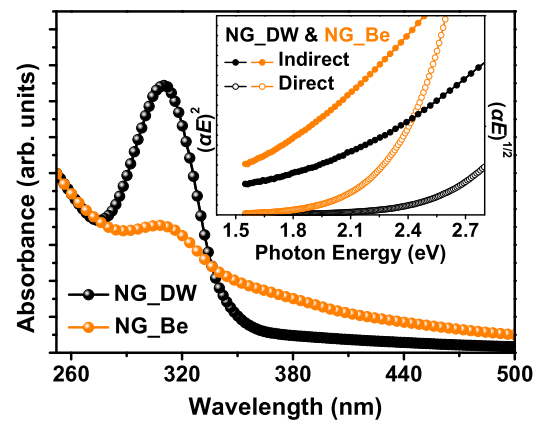

c

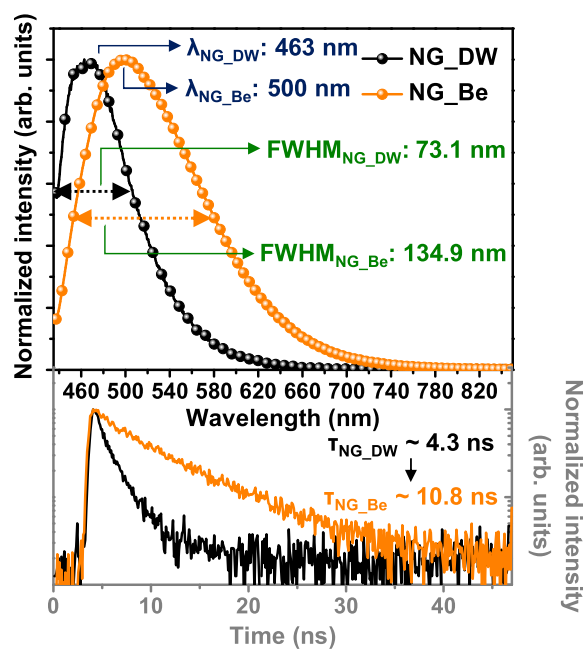

e

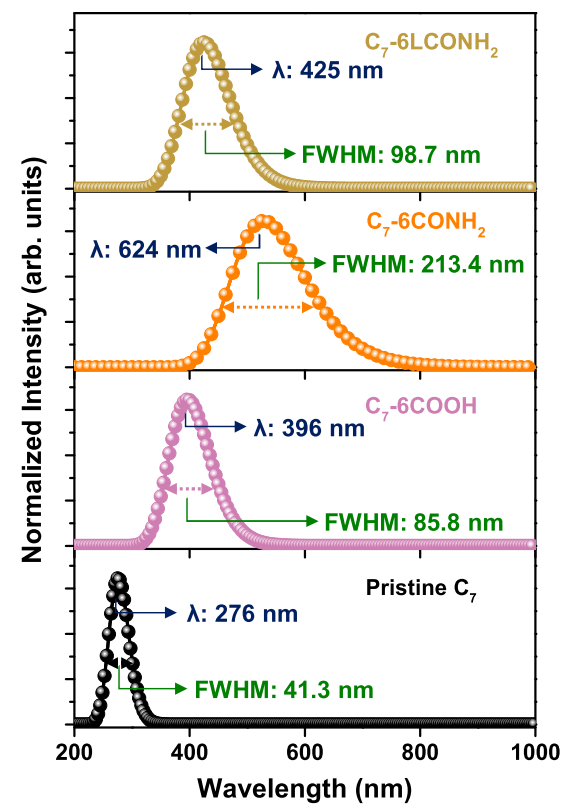

Fig. 14. These shifts can be ascribed to changes in the $f$ values of the electronic transitions caused by the distorted molecular symmetry as well as the different electron distribution in the graphene moieties ${ }^{26}$.

Specifically, depending on the oscillator strengths of $S_{0} \rightarrow S_{n}$ for $\mathrm{C}_{7}$-GQDs in Fig. $4 \mathrm{~b}$, the enhanced absorption capacity at longer wavelengths $(350-500 \mathrm{~nm})$ is significantly affected by the b

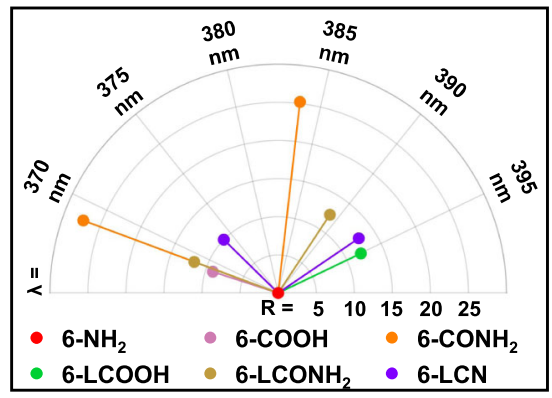

d

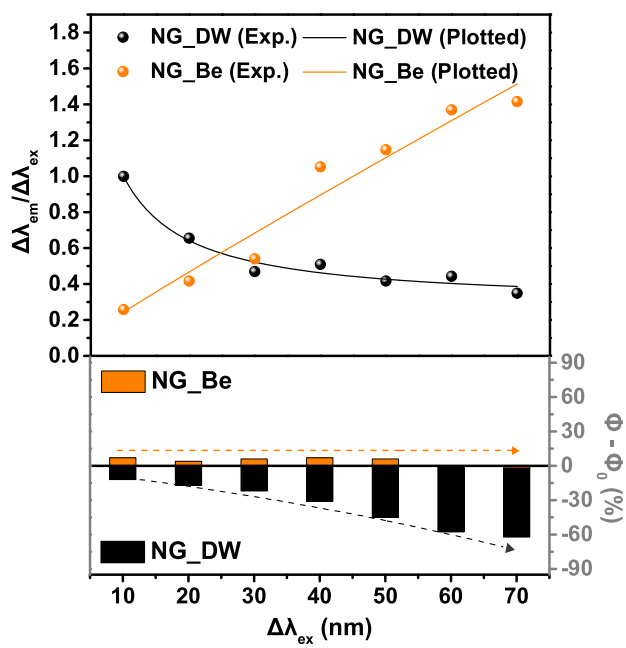

f
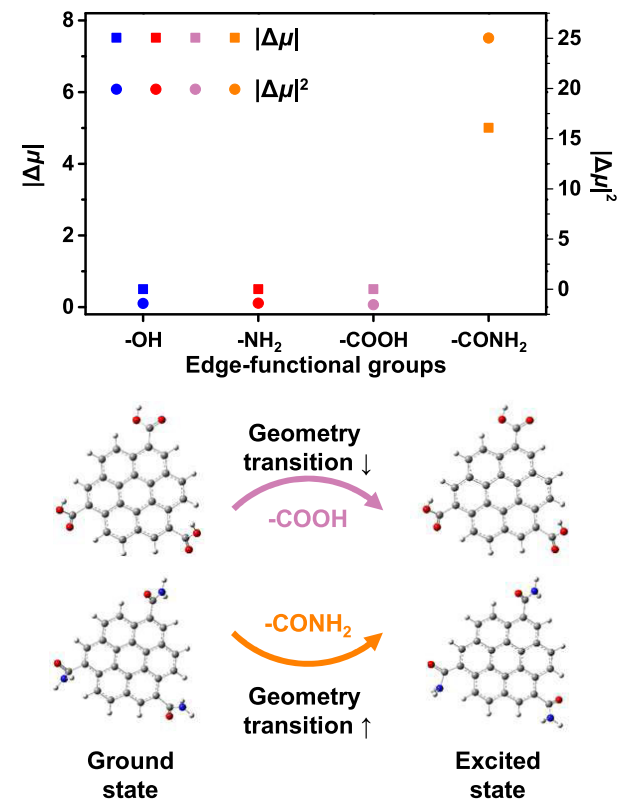

$\mathrm{N}$-related surface states. In particular, according to the frontier molecular orbital (MO) and natural transition orbital (NTO) calculations in Supplementary Figs. $15-18$, the $-\mathrm{CONH}_{2}$ functional group at the edge of the NGs can induce an obvious decrease in the electron density delocalization in the HOMO and LUMO as well as spatial HOMO-LUMO separation, resulting from its highly distorted molecular structure ${ }^{26}$. Additionally, the 
Fig. 4 Experimental and theoretical investigations on the optical properties of NGs. a UV-vis absorption spectra of NG_DW and NG_Be (top). Two excitonic absorption peaks: at $<270 \mathrm{~nm}$ by the $\pi \rightarrow \pi^{\star}$ transition of aromatic sp $p^{2}$ domains, at $310 \mathrm{~nm}$ by the $\mathrm{n} \rightarrow \pi^{\star}$ transition of $\mathrm{O}$ - and $\mathrm{N}$-containing bonds ${ }^{48}$. (inset: plots of $(\alpha E)^{2}$ and $(\alpha E)^{1 / 2}$ against photon energy $(E)$ for the two types of NG solutions $\left(\alpha\right.$ : absorbance)). $\mathbf{b}$ Calculated $f$ of $C_{7}-G Q D s$ with different types of edge-functional groups. The resulted magnitude of $f$-values is represented by the radius $\left(R=10 \log \left(f / f_{6-N H 2}\right)\right)$ and absorption wavelength is represented by the angular variation. $\mathbf{c}$ PL (top) and TRPL (bottom) spectra of NGs. d Comparison of emission peak positions (top) and QYs (bottom) of NGs as a function of $\lambda_{\text {ex }}$. e Theoretically simulated PL spectra of $C_{7}-G Q D s$ with different types of edge-functional groups. $\mathbf{f}$ Theoretical calculation of ground and excited-state dipole moments of the $\mathrm{C}_{7}-\mathrm{GQDs}$ with different edge-functional groups (top). Frontier molecular orbitals for $\mathrm{C}_{7}-6 \mathrm{COOH}$ and $\mathrm{C}_{7}-6 \mathrm{CONH}_{2}$ at the optimized geometries (bottom).

existence of a self-passivated $\pi$-linker, namely, a vinyl bridge, can provide a much larger $\pi$-conjugation domain to ensure effective $\pi$ delocalization with strong extended intramolecular charge transfer ${ }^{27}$. From these observations, the disruption of the structural symmetry or elongation of the $\pi$-conjugation was revealed to cause splitting in the $\pi$ and $\pi^{*}$ levels with a decrease in the energy gap between the HOMO and LUMO, as shown in Supplementary Fig. 19, leading to a broadening of the absorption band towards the longer wavelength region ${ }^{28}$.

The corresponding PL spectra of each NG are presented in Supplementary Fig. 24, exhibiting a strong dependence on the types of edge-functional moieties. In detail, as shown in Fig. 4c, d, and Supplementary Figs. 24-26, there are different PL characteristics in terms of peak wavelength, FWHM, quantum yield (see "Supplementary Discussion 3"), and lifetime depending on the type of NGs. Based on the abovementioned chemical composition and structural analyses, these observations are assumed to be an outcome of the combined and interactive effects of (1) internal charge transitions and (2) solvation dynamics, which are attributed to the changes in the edge or surface states induced by the heteroatom-doping profile.

First, the overall photoluminescence (PL) features of each NG in Fig. $4 \mathrm{c}$ and $\mathrm{d}$ are ascribed to a disruption of the structural symmetry or elongation of the $\pi$-conjugation caused by the presence of functional groups on the material surfaces. On the basis of our calculations, the introduction of edge-functional moieties in NGs can induce substantial redshifts of their emission characteristics, as shown in Fig. 4e, which are associated with the modifications of their inter- and intra-band electron transition processes ${ }^{29-31}$.

Another cause of the difference in $\lambda_{\text {ex }}$-dependent PL characteristics seen at top of Fig. $4 \mathrm{~d}$ originates from the correlation between molecular configuration (chemical and geometrical features) and the dipole moment of the molecules ${ }^{32,33}$. According to the Lippert equation (see "Supplementary Note 1") 32 , such phenomena can be explained by the solvent relaxation and dipolar reorganization effect of the vicinal solvent molecules, where the solvent effect governs changes in the dipole moment (fluorophore) under PL excitation ${ }^{34}$.

As mentioned above, the presence of $-\mathrm{CONH}_{2}$ functional groups at the surface of NGs can lead to strong intramolecular charge migration, resulting in a maximum change in the dipole moment between the ground and excited states among various chemical configurations (Fig. 4f). Note that, for simplicity, the chain length effect was omitted from the estimation of the dipole moment induced by edge-functional groups. At this point, to reduce the interaction between the excited state dipole and the solvent molecules induced by this transition, the solvent cage surrounding the QD surface is inevitably relaxed, which causes both inhomogeneous broadening and a redshift of the PL spectra.

Photocatalytic degradation test and analysis. In conjunction with all of the above confirmation of consistency between our theoretical predictions and the experimental results, the effectiveness was verified to strengthen the feasibility of our work in that, by manipulation of the QD's inherent characteristics, their practical uses can be expanded. In terms of our proposed platform for diverse opto-chemical applications, we monitored NGassisted photocatalytic degradation of rhodamine $\mathrm{B}(\mathrm{RhB})$ and uric acid (UA), where photodecomposition in the latter case can potentially be used for the in vivo cure of metabolic diseases, e.g., Lesch-Nyhan syndrome, gout, and cardiovascular disease $\mathrm{s}^{35,36}$.

For the photocatalytic test, upon exposure to a broadband visible light source ( $400 \mathrm{~nm}$ longpass filtered xenon lamp, $38.6 \mathrm{~mW} \cdot \mathrm{cm}^{-2}$ ), temporal degradation profiles of both target molecules (in aqueous media), which were physically adsorbed onto the surface of either the NGs (shell) or the $\mathrm{TiO}_{2} \mathrm{NPs}$ (core), were measured. As a pre-estimation of the above catalytic effects, possible initial degradation sites in each pollutant (marked with red circles in Fig. 5a) were theoretically elucidated by calculations involving Fukui functions $\left(f^{0}\right)$ and accordingly resulted in values of local softness $\left(s^{0}\right)$ for radical attack ${ }^{37,38}$, where higher photodegradation efficiency for $\mathrm{RhB}$ is expected from its higher $s^{0}$ value. Additionally, the number of radicals generated during photocatalysis strongly reflects the light absorption capacity of the catalysts (more description in Supplementary Discussion 4), and among the different types of NGs in our study, NG_Be is expected to exhibit the highest catalytic activity for the degradation of organic pollutants (Fig. 4a).

In the experimental results shown in Fig. $5 c$, d, similar trends of photocatalytic effects were observed, where the pseudo-firstorder rate constants $(k)$ of the degradation curves with $\mathrm{TiO}_{2} @ \mathrm{NG} \_\mathrm{Be}$ were found to be 0.269 and $0.193 \mathrm{hr}^{-1}$ for $\mathrm{RhB}$ and UA, respectively. In contrast, when NG_DW was used as a shell material, as predicted by the above theoretical analyses, the decay constants of degradation decreased slightly to 0.0929 and $0.0548 \mathrm{hr}^{-1}$ due to the lower light-harvesting capacitance of this material. Remarkably, when pure $\mathrm{TiO}_{2}$ NPs were used for the removal of $\mathrm{RhB}$, the decay constant was as low as $k=0.0179 \mathrm{hr}^{-1}$ (Supplementary Fig. 27) due to the wide intrinsic band gap ${ }^{39}$, but, on the other hand, the core-shell NPs fabricated by introducing only $0.5 \mathrm{wt} \% \mathrm{NGs}$ onto the $\mathrm{TiO}_{2}$ surface could substantially enhance the rate of photocatalytic activity up to $\sim 16$ times. Considering that the influence of NGs incorporated into the surface of $\mathrm{TiO}_{2}$ on its morphology and phase structure is negligible, the enhanced photocatalytic activity can be attributed to the photo- and electrochemical properties of the materials.

With respect to charge kinetics, the mechanism of the above enhancement can also be elucidated by the synergistic effect of quaternary $\mathrm{N}\left(\mathrm{N}_{\mathrm{q}}\right)$ and pyridinic $\mathrm{N}\left(\mathrm{N}_{\text {pydi }}\right)$, as shown in Fig. $5 \mathrm{~b}$. Specifically, $\mathrm{N}_{\mathrm{q}}$ can serve as an electron transfer facilitator for the fast and effective transfer of photogenerated electrons to the photodegradation active site because it aids in supporting electron delocalization effects without destroying $s p^{2}$-hybridized graphitic structures ${ }^{40}$. On the other hand, $\mathrm{N}_{\text {pydi }}$ at the edges or defects can play a dual functional role as a strong electron-withdrawing group, which results in a more negative electrostatic potential (ESP) charge, as well as a catalytic active site for the removal of organic pollutants ${ }^{41}$. Furthermore, the $\mathrm{C}$ atoms in the vicinity of $\mathrm{N}_{\text {pydi }}$ can easily capture $\mathrm{H}_{2} \mathrm{O}$ and $\mathrm{O}_{2}$ molecules with high $s^{+}$and subsequently form the catalytic molecules $\bullet \mathrm{OH}^{-}$and $\bullet \mathrm{O}_{2}{ }^{-}$. 
a

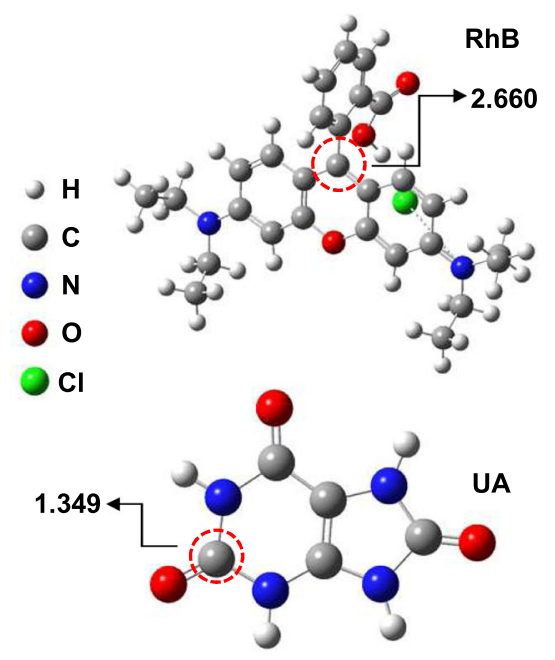

C
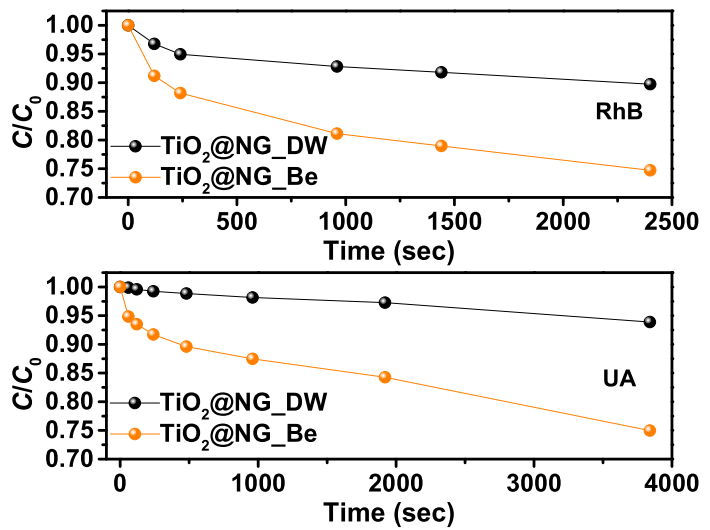

e

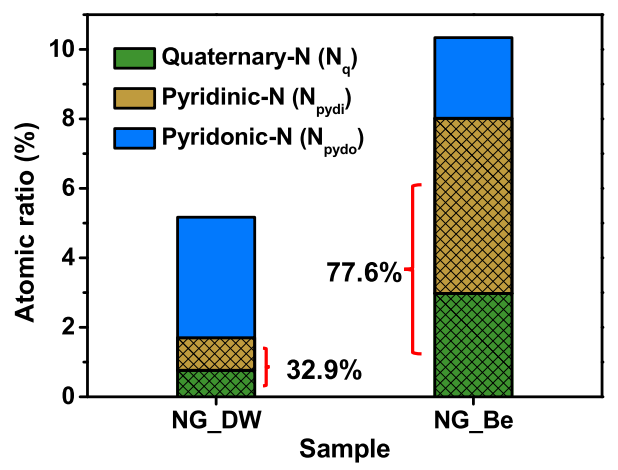

b

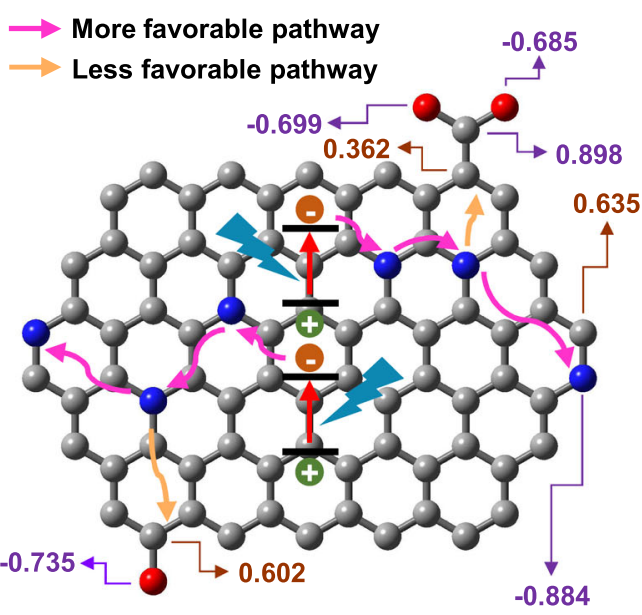

d
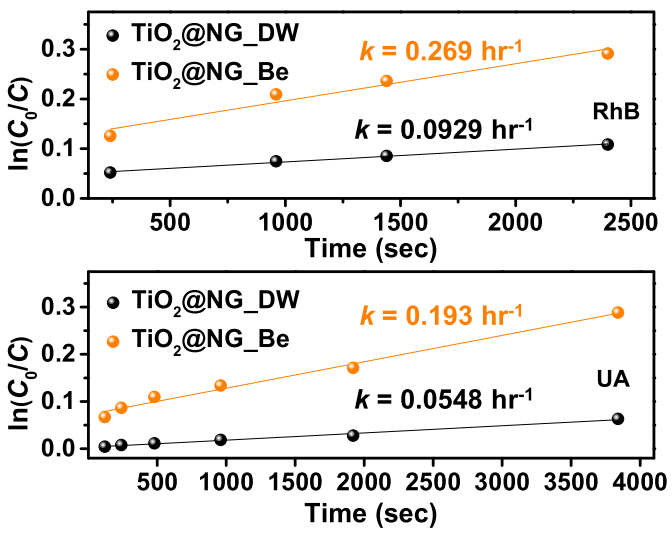

$\mathbf{f}$

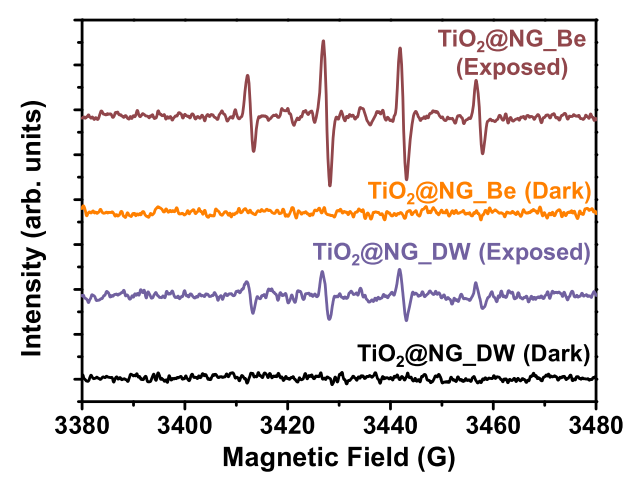

Fig. 5 Photocatalytic activity of NG-decorated $\mathrm{TiO}_{2}$ composites. a Optimized chemical structures of RhB (top) and UA (bottom). Their local softness values $\left(s^{0}\right)$ were calculated using the NBO analysis. b Calculated local softness values ( $s^{+}$in brown) of the N-doped graphitic carbon nanostructure. Atomic charges in purple were obtained from the ESP fitting according to the Merz-Kollman approach at the B3LYP/6-311 + G(d) level. c Photocatalytic degradation of RhB (top) and UA (bottom) in the presence of $\mathrm{TiO}_{2} @ \mathrm{NG}_{-} \mathrm{DW}$ and $\mathrm{TiO}_{2} @ \mathrm{NG} \_$Be catalysts under visible light irradiation $(\lambda>400 \mathrm{~nm}$, $38.6 \mathrm{~mW} \mathrm{~cm}^{-2}$ ). d First-order kinetics of RhB (top) and UA (bottom) photocatalytic degradation by different photocatalysts. e Chemical compositions of $\mathrm{N}$-containing groups in two types of NGs, obtained from N 1s XPS spectra. $\mathbf{f}$ EPR spectra of photocatalyst in aqueous dispersion for $\mathrm{DMPO}^{-\bullet \mathrm{OH}^{-}}$under dark and visible light irradiation conditions. 
From another perspective, substituting $\mathrm{N}$ atoms in the basal plane of NGs is known to induce the formation of n-type domains, which are expected to facilitate the free charge carrier distribution of NGs, which is attributed to the coexistence of pand n-type domains in the QDs ${ }^{42}$. Additionally, due to their nanoscale size, this configuration substantially leads to changes in the near-surface electric field, which has a significant influence on intramolecular photoinduced charge separation (Supplementary Note 2). Accordingly, $\mathrm{p}$ - and n-type regions in graphitic structures can be regarded as an important factor in improving exciton separation and carrier migration efficiencies in NGassisted photocatalysis systems. As shown in Fig. 5e and Supplementary Fig. 8, each NG has a different $\mathrm{N}$ content and $\mathrm{N}$-bonding configuration. NG_Be contains the largest amount of both $\mathrm{N}_{\mathrm{q}}$ and $\mathrm{N}_{\text {pydid }}$, as well as the highest $\mathrm{N} / \mathrm{C}(0.14)$ and $\mathrm{N} / \mathrm{O}$ (0.63) atomic ratios, implying that $\mathrm{TiO}_{2} @ \mathrm{NG}_{-} \mathrm{Be}$ may show not only the highest internal charge dissociation efficiency of photogenerated hole-electron pairs but also the highest generation rate of the free radicals.

To verify these hypotheses, an electron paramagnetic resonance (EPR) spin-trap measurement was performed by using DMPO (5,5-dimethyl-1-pyrroline- $N$-oxide) as a spin-trapping agent. As shown in Fig. $5 \mathrm{f}$ and Supplementary Fig. 28, no DMPO- $\mathrm{OH}$ spin adducts were observed in the dark for either $\mathrm{TiO}_{2} @ \mathrm{NG} \_\mathrm{DW}$ or $\mathrm{TiO}_{2} @ \mathrm{NG} \_\mathrm{Be}$, whereas the characteristic signals for DMPO- ${ }^{\circ} \mathrm{OH}$ (1:2:2:1 quartet pattern) emerged under visible light irradiation. More importantly, it can be clearly seen that the $\cdot \mathrm{OH}$ signal of $\mathrm{TiO}_{2} @ \mathrm{NG}_{-} \mathrm{Be}$ is much stronger than that of $\mathrm{TiO}_{2} @ \mathrm{NG}$ DW. From these observations, we can conclude that the chemical configurations of NGs could modulate the generation rate of active radicals for the photocatalytic degradation of organic pollutants.

In summary, we developed a "creative" approach to synthesize customizable NGs with a highly ordered graphitic structure via the one-step thermolytic self-assembly reaction. Oxidative activation of the nitrile groups in $\mathrm{FN}$ is effectively controlled by the type of solvent, leading to changes in the elemental compositions (N/C and $\mathrm{N} / \mathrm{O}$ ratios) and N-bonding configurations $\left(\mathrm{N}_{\mathrm{q}}, \mathrm{N}_{\text {pydi }}, \mathrm{N}_{\mathrm{am}}\right.$ and $\mathrm{N}_{\text {pydo }}$ ) of NGs. The differences in the chemical configurations of NGs strongly influenced their optoelectronic behaviours as well as their surface morphology features.

Using various computational methods, in regards to the kinetics and thermodynamics of FN activation, we systemically confirmed the solvent catalytic effects on the formation of NGs. As another verification of our reaction mechanism, we also investigated the correlation between the structural features and optical characteristics of NGs.

Finally, it was clearly observed that the introduction of NGs onto the surface of $\mathrm{TiO}_{2}$ has little influence on its structure and morphology, but it significantly enhances the efficiency of light harvesting, as well as charge separation of the photocatalyst due to their N-related chemical components. From these findings, our future studies are expected to provide a variety of innovations in the field of opto-electronics, biological applications, and chemical sensors.

\footnotetext{
Methods

Preparation of NGs. NGs were synthesized by thermal treatment of FN powder purchased from the Tokyo Chemical Industry. $0.1 \mathrm{~g}$ of FN was dispersed in $10 \mathrm{~mL}$ of each solvent (DI-water, tetrahydrofuran, butyraldehyde, and benzaldehyde), injected into a $100 \mathrm{~mL}$ Teflon liner, and then heated to $170{ }^{\circ} \mathrm{C}$ for $10 \mathrm{~min}$ in a stainless-steel reactor using a hot plate while stirring continually to obtain stable and uniformed products. The colours of the liquid consequently changed from colourless to pale yellow (NG_DW), normal yellow (NG_THF), dark yellow $\left(\mathrm{NG} \_\mathrm{Bu}\right)$, and dark red (NG_Be), indicating the successful formation of NGs. After
}

the excess solvent was removed by vacuum evaporator (or vacuum oven), the final products (NGs) were recovered by freeze-drying process.

Instrumentation and measurements. AFM imaging was performed in the air using an NX-10 instrument (Park systems). Cs-corrected STEM (Cs-corrected STEM) images of NGs were obtained with a JEM-ARM200F operated at an accelerating voltage of $80 \mathrm{kV}$ and equipped with a cold field emission gun (CFEG). UV-vis absorption and PL spectra were acquired by a Jasco V-670 spectrophotometer and a Horiba NanoLog-C, respectively. FT-IR spectra were obtained by an FT-IR spectrophotometer (Jasco FT/IR-6600). ${ }^{1} \mathrm{H}$ NMR spectra were recorded in $\mathrm{CDCl}_{3}$ at $600 \mathrm{MHz}$ (Agilent $600 \mathrm{MHz}$ Premium COMPACT NMR Magnet). Raman spectra were obtained using a Horiba Jobin Yvon-Labram HR UV-VisibleNIR Raman microscope spectrometer. NEXAFS measurements were conducted at the soft X-ray 10D XAS KIST (Korea Institute of Science and Technology) beamline. Each spectrum of NGs was obtained in total electron yield mode at room temperature and $\sim 10^{-8}$ Torr, and they were then normalized with respect to the incident photon flux. All spectra were acquired at an incidence angle of $54.7^{\circ}$, where the peak intensities are independent of the angular symmetry dependence. The $\pi^{*}$ resonance is representative of the unsaturated $\mathrm{C}=\mathrm{C}$ or graphitic $\left(s p^{2}\right)$ bond above and below the plane, including the $\mathrm{C}-\mathrm{N}$ transitions $\left(\mathrm{CK}_{1}, 285.4 \mathrm{eV}\right)$, while the in-plane $\sigma^{*}$ feature is typical of a C-C bond ${ }^{43,44}$. The four resonances in the intermediate region between the $\pi^{*}$ and $\sigma^{*}$ features were observed at $\sim 287.2 \mathrm{eV}$ $\left(\mathrm{CK}_{2}\right), \sim 288.3 \mathrm{eV}\left(\mathrm{CK}_{3}\right), \sim 289.1 \mathrm{eV}\left(\mathrm{CK}_{4}\right)$, and $\sim 289.5 \mathrm{eV}\left(\mathrm{CK}_{5}\right)$ and were assigned to functional groups $\left(\mathrm{CK}_{2}: \mathrm{C}-\mathrm{O}, \mathrm{CK}_{3}: \mathrm{C}=\mathrm{O}, \mathrm{CK}_{4}: \mathrm{O}-\mathrm{C}=\mathrm{O}\right.$ and $\mathrm{CK}_{5}$ : various amide groups) that decorate the basal planes and the edge sites of $\mathrm{NGs}^{45}$. XPS analysis was carried out using a monochromated $\mathrm{Al} \mathrm{Ka} \mathrm{X-ray} \mathrm{source}(h v=1486.6 \mathrm{eV})$. To measure the electron lifetime of NGs, TRPL spectroscopy was employed. The second harmonic generation $(\mathrm{SHG}=370 \mathrm{~nm})$ of a tunable Ti:sapphire laser (MaiTai, Spectra-Physics) with an $\sim 100$ fs pulse width and $80 \mathrm{MHz}$ repetition rate was used as the excitation source. EPR measurements were performed using a CWEPR (microwave frequency and power of $9.64 \mathrm{GHz}$ and $3 \mathrm{~mW}$, respectively, and modulation frequency of $100 \mathrm{kHz}$ ).

Preparation of $\mathbf{T i O}_{\mathbf{2}} @$ NG powders. The powders were prepared by a mild temperature hydrothermal process to avoid further oxidation of NGs. Typically, $\mathrm{TiO}_{2}$ (Aeroxide ${ }^{\circledR}$ P25, $20 \mathrm{mg}$ ) was added to $10 \mathrm{~mL}$ of $\mathrm{NG}$ aqueous solution $(0.01 \mathrm{mg} /$ $\mathrm{mL}$ ), which was then incubated for $24 \mathrm{hr}$ at $70^{\circ} \mathrm{C}$ to decorate $\mathrm{NGs}$ on the $\mathrm{TiO}_{2}$ surface (agitation speed: $400 \mathrm{rpm}$ ); excess DI-water was removed by utilizing a vacuum evaporator. The final products $\left(\mathrm{TiO}_{2} @ \mathrm{NGs}\right)$, which contain 0.5 wt $\%$ of NGs, were collected after the drying process.

Photocatalytic degradation test. Photocatalytic activities of $\mathrm{TiO}_{2} @ \mathrm{NG}$ powders were evaluated by monitoring the degradation of $\mathrm{RhB}$ and UA under the illumination of $38.6 \mathrm{~mW} / \mathrm{cm}^{2}$ of white light ( $<400 \mathrm{~nm}$ cut-off). The photocatalytic experiments were carried out at room temperature. In order to investigate the photodegradation efficiency of the catalyst, the aqueous solutions before/after the photodegradation reaction were analysed by absorption and fluorescence techniques as a function of reaction time.

TD-DFT calculations. The vertical excitations of the lowest five excited states were calculated using TD-DFT with CAM-B3LYP/6-31 + G(d) basis set. To clearly describe the diffuse excited states, we chose a double zeta basis set including polarization and diffuse functions, $6-31+\mathrm{G}(\mathrm{d})$. The ground state geometries of the models were optimized at B3LYP (or CAM-B3LYP)/6-31 + G(d) level of theory and confirmed by frequency calculations $(\mathrm{Opt}+\mathrm{Freq})$. Also, the relaxation of the geometries in S1 was performed at TDDFT-CAM-B3LYP/6-31 + G(d) level of theory. The solvent (solvent: ethanol) is included based on the Polarizable Continuum Model (PCM). The optical bandgaps of each model were calculated to be the difference of energy between the ground and the first excited states. The atomic charges in the ground and the first excited states were obtained using the natural bond orbital (NBO) method. All calculations were carried out by Gaussian 16 software package. First, we modelled and simulated several local structures of NGs with a various number of aromatic rings ( $\mathrm{C}_{x}$-NGs, $x=1,2,4,7,19$, and 36), including different heteroatom functional groups at the edge sites. For the accurate but cost-efficient calculations, we finally selected pristine and edge-functionalized $C_{7}$-GQDs.

\section{Data availability}

The authors declare that support the findings of this study are available within the article and Supplementary Information files, and the extra files are also available from the corresponding author (sbae@kist.re.kr, response timeframe: 1 week) upon reasonable request. The data are not publicly available due to privacy or ethical restrictions. Also, the raw/processed data required to reproduce these findings cannot be shared at this time as the data also form part of an ongoing study.

Received: 28 January 2021; Accepted: 10 September 2021; Published online: 07 October 2021 


\section{References}

1. Hutton, G. A. M., Martindale, B. C. M. \& Reisner, E. Carbon dots as photosensitisers for solar-driven catalysis. Chem. Soc. Rev. 46, 6111-6123 (2017).

2. Li, X., Rui, M., Song, J., Shen, Z. \& Zeng, H. Carbon and graphene quantum dots for optoelectronic and energy devices: A review. Adv. Funct. Mater. 25, 4929-4947 (2015).

3. Chung, S., Revia, R. A. \& Zhang, M. Graphene quantum dots and their applications in bioimaging, biosensing, and therapy. Adv. Mater. 33, 1904362-26 (2021)

4. Yeh, T.-F., Teng, C.-Y., Chen, S.-J. \& Teng, H. Nitrogen-doped graphene oxide quantum dots as photocatalysts for overall water-splitting under visible light illumination. Adv. Mater. 26, 3297-3303 (2014).

5. Wu, X., Ding, S.-J., Lin, K. \& Su, J. A review on the biocompatibility and potential applications of graphene in inducing cell differentiation and tissue regeneration. J. Mater. Chem. B 5, 3084-3102 (2017).

6. Sekiya, R., Uemura, Y., Murakami, H. \& Haino, T. White-light-emitting edgefunctionalized graphene quantum dots. Angew. Chem. Int. Ed. 26, 5619-5623 (2014).

7. Kim, D. et al. Graphene quantum dots prevent $a$-synucleinopathy in Parkinson's disease. Nat. Nanotechnol. 13, 812-818 (2018).

8. Moon, B. J. et al. Multi-functional nitrogen self-doped graphene quantum dots for boosting the photovoltaic performance of BHJ solar cells. Nano Energy 34, 36-46 (2017).

9. Moon, B. J. et al. Facile and purification-free synthesis of nitrogenated amphiphilic graphitic carbon dots. Chem. Mater. 28, 1481-1488 (2016).

10. Lin, L. et al. A facile synthesis of highly luminescent nitrogen-doped graphene quantum dots for the detection of 2,4,6-trinitrophenol in aqueous solution. Nanoscale 7, 1872-1878 (2015).

11. $\mathrm{Wu}, \mathrm{X}$. et al. Fabrication of highly fluorescent graphene quantum dots using L-glutamic acid for in vitro/in vivo imaging and sensing. J. Mater. Chem. C. 1, 4676-4684 (2013).

12. Tian, R. et al. Facile preparation and the stepwise formation mechanistic investigation of gram-scale nitrogen-doped graphene quantum dots. J. Mater. Chem. C. 5, 9174-9180 (2017).

13. Dong, X. et al. Fast one-step synthesis of N-doped carbon dots by pyrolyzing ethanolamine. J. Mater. Chem. C. 2, 7477-7481 (2014).

14. Jeon, S.-J. et al. Modulating the photocatalytic activity of graphene quantum dots via atomic tailoring for highly enhanced photocatalysis under visible light. Adv. Funct. Mater. 26, 8211-8219 (2016).

15. Rajender, G., Goswami, U. \& Girl, P. K. Solvent dependent synthesis of edgecontrolled graphene quantum dots with high photoluminescence quantum yield and their application in confocal imaging of cancer cells. J. Colloid Interface Sci. 541, 387-398 (2019).

16. Song, Y. et al. Investigation from chemical structure to photoluminescent mechanism: a type of carbon dots from the pyrolysis of citric acid and an amine. J. Mater. Chem. C. 3, 5976-5984 (2015)

17. Wu, Y. et al. White-light-emitting melamine-formaldehyde microspheres through polymer-mediated aggregation and encapsulation of graphene quantum dots. Adv. Sci. 6, 1801432-10 (2019).

18. Yan, Y. et al. van der Waals heterojunction between a bottom-up grown doped graphene quantum dot and graphene for photoelectrochemical water splitting. ACS NANO 14, 1185-1195 (2020).

19. Hasan, M. T. et al. Photo-and electroluminescence from nitrogen-doped and nitrogen-sulfur codoped graphene quantum dots. Adv. Funct. Mater. 28, 1804337-10 (2018)

20. Hao, Y.-N., Guo, H.-L., Tian, L. \& Kang, X. Enhanced photoluminescence of pyrrolic-nitrogen enriched graphene quantum dots. RSC Adv. 5, 43750-43755 (2015).

21. Qu, D. et al. Highly luminescent S, N co-doped graphene quantum dots with broad visible absorption bands for visible light photocatalysts. Nanoscale 5, 12272-12277 (2013).

22. Tang, L. et al. Deep ultraviolet photoluminescence of water-soluble selfpassivated graphene quantum dots. ACS NANO 6, 5102-5110 (2012).

23. Paraja, M. \& Matile, S. Primary anion $-\pi$ catalysis of epoxide-opening ether cyclization into rings of different sizes: Access to new reactivity. Angew. Chem. Int. Ed. 59, 6273-6277 (2020).

24. Vdovenko, S. I. \& Gerus, I. I. Solvent influence on reaction mechanism of the nucleophilic substitution of $\beta$-substituted alkoxyvinyl trifluoromethyl ketones with piperidine. J. Phys. Org. Chem. 21, 279-285 (2008).

25. Peng, J. et al. Graphene quantum dots derived from carbon fibers. Nano Lett. 12, 844-849 (2012)

26. Kothavale, S., Lee, K. H. \& Lee, J. Y. CN-modified imidazopyridine as a new electron accepting unit of thermally activated delayed fluorescent emitters. Chem. Eur. J. 26, 845-852 (2020).

27. Garcia, G., Adamo, C. \& Ciofini, I. Evaluating push-pull dye efficiency using TD-DFT and charge transfer indices. Phys. Chem. Chem. Phys. 15 20210-20219 (2013)
28. $\mathrm{Lu}$, J. et al. D- $\pi-\mathrm{A}$ structured porphyrins for efficient dye-sensitized solar cells. J. Mater. Chem. A 1, 10008-10015 (2013).

29. Martin, E., Weigand, R. \& Pardo, A. Solvent dependence of the inhibition of intramolecular charge-transfer in $\mathrm{N}$-substituted 1,8-naphthalimide derivatives as dye lasers. J. Lumin. 68, 157-164 (1996).

30. Herbich, J. \& Kapturkiewicz, A. Radiative and radiationless depopulation of the excited intramolecular charge transfer states: Aryl derivatives of aromatic amines. Chem. Phys. 158, 143-153 (1991).

31. Bae, H. J. et al. Through-space charge transfer and emission color tuning of dio-carborane substituted benzene. Dalton Trans. 43, 4978-4985 (2014).

32. Bianco, A. et al. Light-induced dipole moment modulation in diarylethenes: a fundamental study. Phys. Chem. Chem. Phys. 18, 31154-31159 (2016).

33. Hovey, R. J. \& Martell, A. E. Dipole moments of metal chelate compounds. IV. metal chelates of analogs and polar substituted analogs of bisacetylacetoneethylenediimine. J. Am. Chem. Soc. 82, 2697-2700 (1960).

34. Mukherjee, S., Chattopadhyay, A., Samanta, A. \& Soujanya, T. Dipole moment change of NBD group upon excitation studied using solvatochromic and quantum chemical approaches: Implications in membrane research. J. Phys. Chem. 98, 2809-2812 (1994).

35. Rock, K. L., Kataoka, H. \& Lai, J.-J. Uric acid as a danger signal in gout and its comorbidities. Nat. Rev. Rheumatol. 9, 13-23 (2013).

36. Feig, D. I., Kang, D.-H. \& Johnson, R. J. Uric acid and cardiovascular risk. N. Engl. J. Med. 359, 1811-1821 (2008).

37. Yang, W., Parr, R. G. \& Pucci, R. Electron density, Kohn-Sham frontier orbitals, and Fukui functions. J. Chem. Phys. 81, 2862-2863 (1984).

38. Yang, W. \& Parr, R. G. Hardness, softness, and the Fukui function in the electronic theory of metals and catalysis. Proc. Natl Acad. Sci. USA 82 6723-6726 (1985).

39. Pan, D. et al. Efficient separation of electron-hole pairs in graphene quantum dots by $\mathrm{TiO}_{2}$ heterojunctions for dye degradation. ACS Sustain. Chem. Eng. 3, 2405-2413 (2015)

40. Wang, H. B., Maiyalagan, T. \& Wang, X. Review on recent progress in nitrogen-doped graphene: synthesis, characterization, and its potential applications. ACS Catal. 2, 781-794 (2012).

41. Guo, D. et al. Active sites of nitrogen-doped carbon materials for oxygen reduction reaction clarified using model catalysts. Science 351, 361-365 (2016).

42. Nozik, A. J. \& Memming, R. Physical chemistry of semiconductor-liquid interfaces. J. Phys. Chem. 100, 13061-13078 (1996).

43. Dennis, R. V. \& Schultz, B. J. Near-edge X-ray absorption fine structure spectroscopy study of nitrogen incorporation in chemically reduced graphene oxide. J. Vac. Sci. Technol. B 31, 041204-041209 (2013).

44. Dubin, S. et al. A one-step, solvothermal reduction method for producing reduced graphene oxide dispersions in organic solvents. ACS Nano 4, 3845-3852 (2010).

45. Lalljie, S. P. D. \& Sandra, P. Practical and quantitative aspects in the analysis of FITC and DTAF amino acid derivatives by capillary electrophoresis and LIF detection. Chromatrographia 40, 519-526 (1995).

46. Cappelli, E. et al. Orientation tendency of PLD carbon films as a function of substrate temperature: a NEXAFS study. Diam. Relat. Mater. 14, 959-964 (2005).

47. Schultz, B. J. et al. X-ray absorption spectroscopy studies of electronic structure recovery and nitrogen local structure upon thermal reduction of graphene oxide in an ammonia environment. RSC Adv. 4, 634-644 (2014).

48. Tetsuka, T., Nagoya, A. \& Asahi, R. Highly luminescent flexible aminofunctionalized graphene quantum dots@cellulose nanofiber-clay hybrids for white-light emitting diodes. J. Mater. Chem. C. 3, 3536-3541 (2015).

\section{Acknowledgements}

This work was financially supported by the Korea Institute of Science and Technology (KIST) Institutional Program, the Ministry of Trade, Industry \& Energy of Korea (20011317), and the National Research Council of Science \& Technology (NST) grant (CRC-20-01-NFRI).

\section{Author contributions}

B.J.M. and S.B. conceived the idea and designed the experiments. B.J.M., S.J.K., A.L. and Y.O. synthesized and characterized the samples. B.J.M. and B.H.H. performed the DFT calculations and analysis. B.J.M. and S.H.L. carried out TRPL measurements. S-K.L. and T-W.K. conducted the NEXAFS measurement. B.J.M. and Y.O. performed photocatalytic experiments. B.J.M., S.J.K., A.L. and S.B. wrote the manuscript and prepared the figures. All authors participated in discussions and commented on the manuscript.

\section{Competing interests}

The authors declare no competing interests. 


\section{Additional information}

Supplementary information The online version contains supplementary material available at https://doi.org/10.1038/s41467-021-26122-0.

Correspondence and requests for materials should be addressed to Sukang Bae.

Peer review information Nature Communications thanks Jun-Jie Zhu and the other, anonymous, reviewer(s) for their contribution to the peer review of this work. Peer reviewer reports are available.

Reprints and permission information is available at http://www.nature.com/reprints

Publisher's note Springer Nature remains neutral with regard to jurisdictional claims in published maps and institutional affiliations. (c) (1) Open Access This article is licensed under a Creative Commons Attribution 4.0 International License, which permits use, sharing, adaptation, distribution and reproduction in any medium or format, as long as you give appropriate credit to the original author(s) and the source, provide a link to the Creative Commons license, and indicate if changes were made. The images or other third party material in this article are included in the article's Creative Commons license, unless indicated otherwise in a credit line to the material. If material is not included in the article's Creative Commons license and your intended use is not permitted by statutory regulation or exceeds the permitted use, you will need to obtain permission directly from the copyright holder. To view a copy of this license, visit http://creativecommons.org/ licenses/by/4.0/.

(C) The Author(s) 2021 